\title{
Complex networks : application for texture characterization and classification
}

\author{
T. Chalumeau* and L. da F. Costa ${ }^{+}$and O. Laligant* and F. Meriaudeau* \\ * Universite de Bourgogne, Le2i Laboratory, 12 rue de la Fonderie 71200, Le Creusot, France \\ + Universidade de Sao Paulo, IFSC-Av. Trabalhador Sao-Carlense, 400 Centro, Sao Carlos (SP), Brasil
}

Received 16th May 2008; accepted 12th March 2009

\begin{abstract}
This article describes a new method and approach of texture characterization. Using complex network representation of an image, classical and derived (hierarchical) measurements, we present how to have good performance in texture classification. Image is represented by a complex networks : one pixel as a node. Node degree and clustering coefficient, using with traditional and extended hierarchical measurements, are used to characterize "organization" of textures.
\end{abstract}

Key Words: Image processing, texture analysis, complex networks.

\section{Introduction}

Texture analysis have important role in numerous application of image processing. Many different approaches to texture analysis have been proposed. Among the most widely used texture measures are those derived from gray level co-occurence matrices or difference histograms, "texture energy" measures obtained by local linear transforms, and features based on multi-channel Gabor filtering or Markov random field model [1, 2].

Introduced recently $[3,4,5]$, complex networks can be adapted to represent the relation and characterization between elements and become appropriate to characterize picture pattern. It is possible to represent an image as a complex network and used tools from texture networks theory to characterize the created image: segmentation [6], texture analysis [7].

This paper overviews our approach, presents in the first part complex networks and image representation, in the second part methods that were used for comparison. The third part exposes complex networks method's results, with the efficiency of hierarchical measurements. The last part concludes with an overview of the obtained results and suggest possibilities for further improvements and complementary work.

Correspondence to: thomas.chalumeau@u-bourgogne.fr

Recommended for acceptance by David Fofi and Ralph Seulin

ELCVIA ISSN: 1577-5097

Published by Computer Vision Center / Universitat Autonoma de Barcelona 


\section{Complex Network And Image Representation}

\subsection{Complex networks and measurements}

A complex network is a set of nodes connected between them. One connection (or edge) between two nodes (or vertices) indicates an interaction between these two edges. Edges can be binary (i.e. presence or absence of connection) or weighted, and directed or not. The present work is limited to non-directed edges. All complex networks can be represented mathematically by a matrix called the adjacency matrix. With a complex network with $N$ nodes, the adjacency matrix $(W)$ have a dimension $N \times N$. The weight of the connection from each node $j$ to each node $i(i, j=1,2 \ldots, N)$ is represented as $W(i, j)$, with null value being assigned in the absence of such a connection. A second matrix $W_{T}$, binary, is also obtained. It contains only the most significant connections. For example, connections wich are greater weight(only elements of $W$ which are greater than or equal to a threshold $T$ are kept); it can be seen in the example in Figure 1. The characterization of the topological and connectivity properties of complex networks can be achieved by using measurements borrowed from graph theory [8] and complex network research [5] including but being by no means limited to:

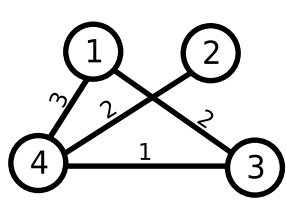

(a)

\begin{tabular}{|l|l|l|l|}
\hline 0 & 0 & 2 & 3 \\
\hline 0 & 0 & 0 & 2 \\
\hline 2 & 0 & 0 & 1 \\
\hline 3 & 2 & 1 & 0 \\
\hline
\end{tabular}

(b)

\begin{tabular}{|l|l|l|l|}
\hline 0 & 0 & 1 & 1 \\
\hline 0 & 0 & 0 & 1 \\
\hline 1 & 0 & 0 & 0 \\
\hline 1 & 1 & 0 & 0 \\
\hline
\end{tabular}

(c)

Figure 1: (a): Weighted small complex network (b): the adjacency matrix $W$, (c): $W_{T}$ matrix (binary) obtain with $T=2$.

Degree: The degree of a given node is equal to the number of connections which it makes. For weighted connections the degree of a node is called strength and corresponds to the sum of all the weights of the respective links. An example in Figure 2 illustrates this definition. The frequency histograms of the degrees provide an important characterization of the connectivity of the network under analysis.

Clustering Coefficient: The clustering coefficient of a given node $i$ is defined as:

$$
C_{i}=\frac{\text { Number of connections between nodes connected to node } i}{\text { Number of possible connections between these nodes }}
$$

whenever the denominator is equal to zero, we impose $C_{i}=0$. Note that it follows that $0<C_{i}<1$ for any possible node. Figure 2 illustrates the calculation of the clustering coefficient for a simple network.

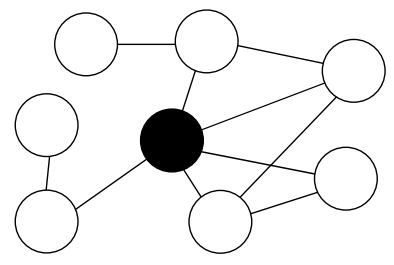

Figure 2: Illustration of degree (D) and clustering coefficient (C) calculation of the node represented in black: $D=5$ and $C=\frac{3}{5 \times 4 \backslash 2}=\frac{3}{10}$. 
Hierarchical Measurements: Several complex networks measurements, including the node degree and clustering coefficient, can be generalized to take into account not only the immediate neighborhood of a node, but also those which are at successive distances (i.e. 2, 3, ...) from that specific node [9] (example in Figure 3). In particular, the hierarchical degree of a node for hierarchical level $i$ corresponds to the number of edges connecting the nodes at distance $i$ to the nodes at distance $i+1$. The hierarchical clustering coefficient of a given node for hierarchical level $i$ is calculated in the same way as the traditional clustering measurement, but considering the edges between the nodes at distance $i$ and the nodes at distance $i+1$.

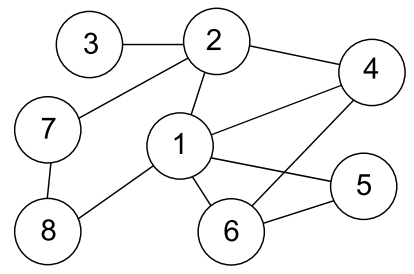

(a)

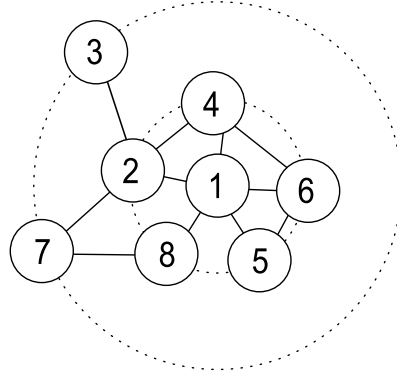

(b)

Figure 3: Hierarchical representation (b) of the complex network (a) for the node 1.

For all measurements, all nodes of the complex network are characterized. To have a global information of the complex network, two parameters are extracted from these histograms : the mean and the standard deviation.

\subsection{Image representation}

To transform an image into a complex network, we assume that each pixel is represented by one node. Weights of edges are defined with the grey level of pixels. The connection between two nodes $i$ and $j$ is defined by :

$$
W(i, j)=\frac{255-|G(i)-G(j)|}{255}
$$

where $G(i)$ represents the grey level of node $i \in[0,255]$. Weights are defined in the [0,1], zero define no connection, and 1 the maximum connection between two nodes.

Connections between edges are defined only inside a circular region of radius $r$ centered on each pixel. The matrix $W_{T}$ is a thresholded matrix. It is a binary matrix where the value 1 define a connection, and 0 not. An example of construction of the two matrices $W$ and $W_{T}$ can be seen in Figure 4 and a complete representation in complex networks of image is illustrated in Figure 5.

\section{Comparatives methods}

The results obtained by using the complex network methodology have been compared to those provided by the co-occurrence matrices introduced by Haralick [10] and by Gabor filters [11,2].

\subsection{Co-occurence features :}

Co-occurrence matrices consider repeated occurrences of some grey level configuration in the texture. A co-occurrence matrix is constructed by observing pairs of pixels separated by a distance $d$ and incrementing the matrix position corresponding to the grey level of both pixels. The value $p(i, j)$ represents the frequency of occurrence of the situation $f\left(x_{1}, y_{1}\right)=i, f\left(x_{2}, y_{2}\right)=j,\left|x_{1}-x_{2}\right|=d$ or $\left|y_{1}-y_{2}\right|=d$ or $\sqrt{\left(x_{2}-x_{1}\right)^{2}+\left(y_{2}-y_{1}\right)^{2}}=d$. 


\begin{tabular}{|l|l|l|}
\hline 10 & 12 & 28 \\
\hline 16 & 25 & 31 \\
\hline
\end{tabular}

(a)

\begin{tabular}{|c|c|c|c|c|c|}
\hline 0 & 2 & 18 & 6 & 15 & 21 \\
\hline 2 & 0 & 16 & 4 & 13 & 19 \\
\hline 18 & 16 & 0 & 12 & 3 & 3 \\
\hline 6 & 4 & 12 & 0 & 9 & 15 \\
\hline 15 & 13 & 3 & 9 & 0 & 6 \\
\hline 21 & 19 & 3 & 15 & 6 & 0 \\
\hline
\end{tabular}

(b)

\begin{tabular}{|l|l|l|l|l|l|}
\hline 0 & 1 & 0 & 1 & 0 & 0 \\
\hline 1 & 0 & 0 & 1 & 0 & 0 \\
\hline 0 & 0 & 0 & 0 & 1 & 1 \\
\hline 1 & 1 & 0 & 0 & 0 & 0 \\
\hline 0 & 0 & 1 & 0 & 0 & 1 \\
\hline 0 & 0 & 1 & 0 & 1 & 0 \\
\hline
\end{tabular}

(c)

Figure 4: (a): part of image in Grey level (b): adgency matrix $(W)$ of the sub-image representation in complex network, (c): $W_{T}$ matrix with threshold $=8$.

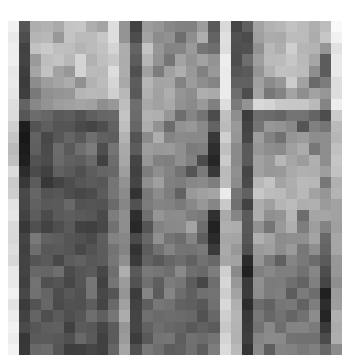

(a)

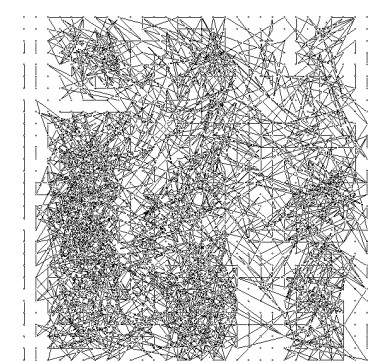

(b)

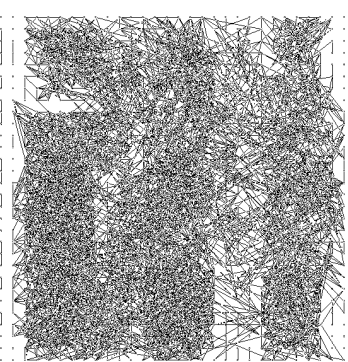

(c)

Figure 5: (a): A $30 \times 30$ sub-image with the $20 \times 20$ usable centered zone (radius $r=5$ ), (b): typical representation of the complex network with threshold $=0.99$, and (c): representation with threshold $=0.98$

Various characteristics can be extracted from the co-occurrence matrix: Energy, Contrast, Correlation, Dissimilarity, Homogeneity. In our case, these characteristics were determined with $d=1$ and $d=5$ [12].

\subsection{Gabor filters :}

Gabor filters which perform a local Fourier analysis, are essentially sine and cosine (complex exponential) modulated by a Gaussian window. In the complex space these filters are expressed as:

$$
h(x, y)=g\left(x^{\prime}, y^{\prime}\right) \cdot e^{j 2 \pi\left(U_{x}+V_{y}\right)}
$$

where $g(x, y)=\frac{2}{2 \pi \lambda \sigma} \cdot e^{-\frac{(x / \lambda)^{2}+y^{2}}{2 \sigma^{2}}}$ and $\left[\begin{array}{c}x^{\prime} \\ y^{\prime}\end{array}\right]=\left[\begin{array}{c}x \cdot \cos (\phi)+y \cdot \sin (\phi) \\ -x \cdot \sin (\phi)+y \cdot \cos (\phi)\end{array}\right]$

$\phi$ is a clockwise rotation along the $x$ axis, $U$ and $V$ represent the frequency coordinates.

$\sigma$ is the standard deviation of the Gaussian envelope (which defines its size) and $\lambda$ is the shape parameter of the Gaussian.

The Gaussian has a circular shape for $\lambda=1$.

The transfer function of $h(x, y)$ is expressed as:

$$
H(x, y)=G\left(u^{\prime}-U^{\prime}, v^{\prime}-U^{\prime}\right)
$$

$$
\text { with } G(u, v)=e^{-2 \pi^{2} \sigma^{2}\left(u^{2} \lambda^{2}+v^{2}\right)} \text { and }\left[\begin{array}{c}
u^{\prime} \\
v^{\prime}
\end{array}\right]=\left[\begin{array}{c}
u \cdot \cos (\phi)+v \cdot \sin (\phi) \\
-u \cdot \sin (\phi)+v \cdot \cos (\phi)
\end{array}\right]
$$

$H(u, v)$ is therefore a Gaussian band-pass filter, which principal axis is oriented at $\phi$ degree from the $u$ axis and with central frequency $F$ defined by : $F=(U+V)^{1 / 2}$ oriented according to the polar angle $\theta$, as shown 


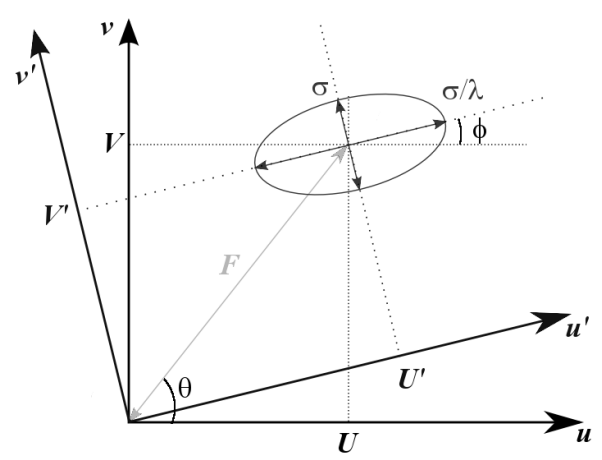

Figure 6: : General Gabor filter in the Fourier space $\theta \neq \phi$.

in Figure 6. In our case, 3 frequency and 6 angles are used, with $\theta=\phi$. Parameter extracted of this results is the energy :

$$
\text { Energy }=\sum \text { pixel }^{2}
$$

\subsection{Wavelets :}

In practice, the 2-D discrete wavelet decomposition can be obtained by using a separable filter bank to the image [13]:

$$
\begin{aligned}
L_{n}\left(b_{i}, b_{j}\right) & =\left[H_{x} *\left[H_{y} * L_{n-1}\right]_{\downarrow 2,1}\right]_{\downarrow 1,2}\left(b_{i}, b_{j}\right) \\
D_{n 1}\left(b_{i}, b_{j}\right) & =\left[H_{x} *\left[G_{y} * L_{n-1}\right]_{\downarrow 2,1}\right]_{\downarrow 1,2}\left(b_{i}, b_{j}\right) \\
D_{n 2}\left(b_{i}, b_{j}\right) & =\left[G_{x} *\left[H_{y} * L_{n-1}\right]_{\downarrow 2,1}\right]_{\downarrow 1,2}\left(b_{i}, b_{j}\right) \\
D_{n 3}\left(b_{i}, b_{j}\right) & =\left[G_{x} *\left[G_{y} * L_{n-1}\right]_{\downarrow 2,1}\right]_{\downarrow 1,2}\left(b_{i}, b_{j}\right)
\end{aligned}
$$

where $*$ denotes the convolution operator, $\downarrow 2,1$ sub sampling along the rows and $\downarrow 1,2$ sub sampling along the columns. $L_{0}$ is the original image. $H$ and $G$ are lowpass and highpass filters.

The decomposition is obtained on $n$ levels by applying recursively the filter bank $\mathrm{n}$ times on the image. Figure 7 shows examples of decomposition for two different levels.
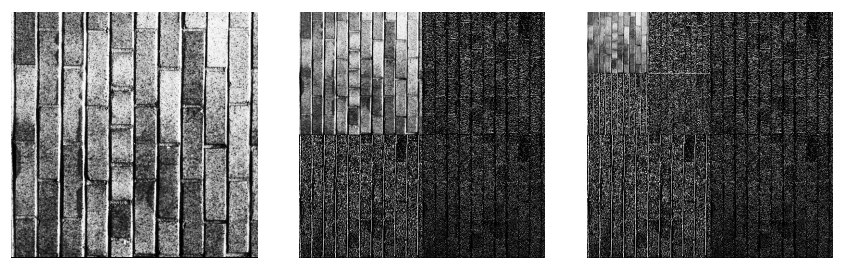

Figure 7: Example of image of texture (a) and 2D discrete wavelet decomposition at level 1(b) and 2(c) with Haar wavelet.

To extract wavelet texture features, the texture is decomposed with the discrete wavelet transform first. Once the image is decomposed, each sub band is characterized by signatures. In our case, the energy signature is 
used. To a sub band, the energy is:

$$
E_{l, d}=\frac{1}{M N} \sum_{i=1}^{M} \sum_{j=1}^{N}\left(I_{l, d}(i, j)\right)^{2}
$$

where $I_{l, d}$ is the coefficient of the $d$ sub image, at the $l$ level of decomposition.

\section{Results}

The comparative study was performed while considering different textures resulting from Brodatz data base. Six different types of textures, illustrated in Figure 8, were used. For all textures, 20 "sub-images" are considered.
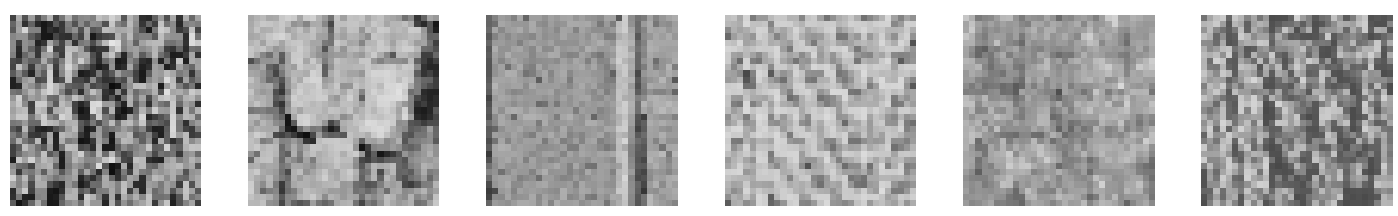

Figure 8: Samples of the 6 classes of textures used.

The classification is performed by a multilayer perceptron, issue of the software TANAGRA [14], with 25 neurons, 500 maximum iterations and a learning rate equal to 0.25 . The method uses $70 \%$ of "sub-images" for training and $30 \%$ for classification. To compare our results of classification with comparative's method, the Error Rate is defined as the number of bad recognitions divided by the number of samples.

Figure 9 shows error rate of classification for our method and Table 1 for comparison methods (only best results of comparative methods are visible). Different experiments was made for complex networks method, with several thresholds and several hierarchical levels. Note that each hierarchical level $k$ in the $x$-axis indicates the use of all hierarchical levels up to $k$, and not just the hierarchical value $k$.

With these results it appears that the use of measurements considering progressive hierarchical levels has a definite effect in improving the classification rate (lower error rate). The minimum of Error Rate is obtained for the level 4 for the threshold equal to 0.75 . The error rate of classification stop to level 5 (defined manually); if higher levels are used, the error rate increase. This is the limit of the hierarchical measurements. This limit is due to the low density or non-existent of connections on higher levels and thus parameters determined (degree and clustering coefficient) can not be good to discriminate classes of networks. This better hierarchical level depends of the image database and the threshold used [7].

\begin{tabular}{|c|c|}
\hline Method & Error Rate \\
\hline co occurence & 0.04 \\
Gabor & 0.4 \\
Wavelet & 0.35 \\
\hline
\end{tabular}

Table 1: Error Rate for comparative methods : Haralick's approach (distance $d=1$ to 5 with 8 angles), Gabor filters( 3 frequencies and 6 angles) and Wavelet(Bior wavelet), using the perceptron classifier .

\section{Conclusion}

Method for texture classification using complex networks had been presented and compared. Two methods for texture classification using complex networks had been presented and compared. ur simple method, us- 


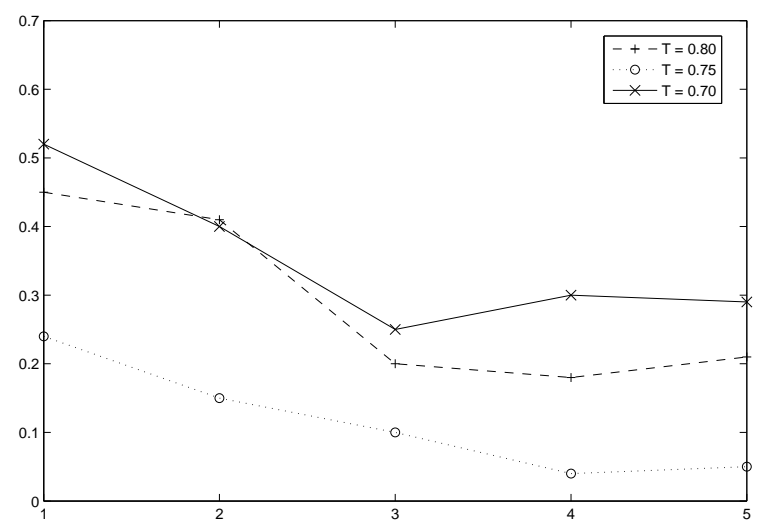

Figure 9: Evolution of Error Rate in function of hierarchical measurements used. Results of classification is obtained by the multilayer perceptron.

ing Complex networks with measurements of topology and connectivity, has a good ability to represent and characterize textures. The interest of hierarchical levels was made and increase the efficiency of the classification of characterization of textures. Although promising results have been obtained, our method used simples parameters (mean and standard deviation).

We are currently working to improve this shortcoming : use more informations about the histograms (i.e. moments and coefficients), an automatic determination of the better hierarchical level to improve the classification. An improvement of the method in relation of the determination of the better threshold can be done using measurements for weighted networks [16].

\section{References}

[1] S. Aksoy and R. Haralick, "Textural features for image database retrieval," Content-Based Access of Image and Video Libraries, 1998. Proceedings. IEEE Workshop on , pp. 45-49, 1998.

[2] P. Kruizinga, N. Petkov, and S. Grigorescu, "Comparison of texture features based on gabor filters," Proceding of the 10th International Conference on Image Analysis and Processing , pp. 142-147, 1999.

[3] M. E. J. Newman, "The structure and function of complex networks," cond-mat/0303516, 2003.

[4] L. da Fontoura Costa, F. A. Rodrigues, G. Travieso, and P. R. V. Boas, "Characterization of complex networks: A survey of measurements," cond-mat/0505185, 2005.

[5] R. Albert and A.-L. Barabasi, "Statistical mechanics of complex networks," cond-mat/0106096, 2001.

[6] L. da Fontoura Costa, "Complex networks, simple vision," cond-mat/0403346 , 2004.

[7] T. Chalumeau, L. da F. Costa, O. Laligant and F. Meriaudeau, "Optimized texture classification by using hierarchical complex networks measurements," Proceding on SPIE Conference Machine Vision Applications in Industrial Inspection XIV, vol. 6383, 2006.

[8] R. Diestel, Graph Theory, Springer, 2nd electronic edition ed., 2000.

[9] L. da Fontoura Costa, "Hierarchical characterization of complex networks," cond-mat/0412761 , 2005. 
[10] L.-K. Soh and C. Tsatsoulis, "Texture analysis of sar sea ice imagery using gray level co-occurrence matrices," IEEE Transactions on geoscience and remote sensing 37(2), pp. 780-795, 1999.

[11] C. Palm and T. M. Lehmann, "Classification of color textures by gabor filtering," Machine GRAPHICS \& VISION Vol. 11, pp 195 219, 2002.

[12] N. Pican, E. Trucco, M. Ross, D. M. Lane, Y. Petillot, and I. Ruiz, "Texture analysis for seabed classification: Co-occurrence matrices vs self-organizingmaps.," IEEE/OES OCEANS '98 Conference Proceedings 1, pp. 424-428, 1998.

[13] G. V. de Wouwer, P. Scheunders, and D. V. Dyck, "Statistical texture characterization from discrete wavelet representations," IEEE Trans. Image Proc. 8, pp. 592-598, April 1999.

[14] R. Rakotomalala, "Tanagra : un logiciel gratuit pour l'enseignement et la recherche," in Actes de EGC'2005, RNTI-E-3 2, pp. 697-702, 2005.

[15] L. da Fontoura Costa and R. M. C. Junior, Shape Analysis and Classification: Theory and Practice, CRC Press, Inc, 2000.

[16] S. Boccaletti and V. Latora and Y. Moreno and M.Chavez and D-U.Hwang, "Complex Networks : Structure and Dynamics" Physics Reports Vol. 424, pp. 175-308, 2006. 